\title{
Effects of Thermal and Humidity Aging on the Interfacial Adhesion of Polyketone Fiber Reinforced Natural Rubber Composites
}

\author{
Han Ki Lee, ${ }^{1}$ Dae Sik Kim, ${ }^{1}$ Jong Sung Won, ${ }^{2}$ Da Young Jin, ${ }^{2}$ \\ Hyun Jae Lee, ${ }^{2}$ and Seung Goo Lee ${ }^{2}$ \\ ${ }^{1}$ New Technology Development Team, Research \& Development Division, Hyundai-ngv, Seoul 151-742, Republic of Korea \\ ${ }^{2}$ Department of Organic Materials Engineering, Chungnam National University, Daejeon 34134, Republic of Korea
}

Correspondence should be addressed to Seung Goo Lee; 1sgoo@cnu.ac.kr

Received 7 August 2016; Accepted 12 October 2016

Academic Editor: Belal F. Yousif

Copyright ( $) 2016$ Han Ki Lee et al. This is an open access article distributed under the Creative Commons Attribution License, which permits unrestricted use, distribution, and reproduction in any medium, provided the original work is properly cited.

Polyketone fiber is considered as a reinforcement of the mechanical rubber goods (MRG) such as tires, automobile hoses, and belts because of its high strength and modulus. In order to apply it to those purposes, the high adhesion of fiber/rubber interface and good sustainability to aging conditions are very important. In this study, polyketone fiber reinforced natural rubber composites were prepared and they were subjected to thermal and humidity aging, to assess the changes of the interfacial adhesion and material properties. Also, the effect of adhesive primer treatment, based on the resorcinol formaldehyde resin and latex (RFL), of polyketone fiber for high interfacial adhesion was evaluated. Morphological and property changes of the rubber composites were analyzed by using various instrumental analyses. As a result, the rubber composite was aged largely by thermal aging at high temperature rather than humidity aging condition. Interfacial adhesion of the polyketone/NR composites was improved by the primer treatment and its effect was maintained in aging conditions.

\section{Introduction}

As general reinforcements of rubber composites, rayon, nylon, and polyester fibers have been used for a long time due to their reasonable performance and usefulness. Recently, application of the para-aramid fiber which has high tenacity and modulus is gradually increasing in order to satisfy high performance requirements including specialty tire and tire weight reduction. However, in working with high performance fibers like para-aramid, it is normally difficult to obtain an excellent adhesion with simple processing and their fatigue resistance is not good $[1,2]$.

Polyketone fiber, recently developed, is available by copolymerization of carbon monoxide and unsaturated hydrocarbon monomer, ethylene. Hence, the cost of its raw material is cheap and the spinning process is environmentally friendly. Also, it has the additional benefits of similar tenacity and modulus to para-aramid and has higher breaking elongation and good adhesion property with a rubber matrix. Therefore, it is expected to be the reinforcement of mechanical rubber goods (MRG) such as tires, hoses, and protective gloves [3].

Adhesion strength between textile cord and rubber matrix can affect the entire performance of a tire. To improve interfacial adhesion between polar cord and nonpolar rubber matrix, an adhesive agent must be used. Since 1935, resorcinol-formaldehyde-latex (RFL) has been used to promote the cord/rubber adhesion. Generally, textile cord must be coated in RFL dip followed by precuring. Low viscosity and good wetting properties have advantages of these aqueous dips. After curing, they are transformed to an insoluble system. The latex gives required flexibility to adhesive agent and reactivity to rubber. Moreover, RF resin provides a desirable heat and fatigue resistance by forming a threedimensional network. At fiber cord/rubber interface, the functional groups of polar cord and unsaturated zones of rubber undergo reactions with methylol and vinyl groups of RFL, respectively. Physical forces originated from interpenetration 
and interlocking of the RF resin with the fiber can be a major factor of adhesion, as well [4-9].

On the other hand, the rubber composites are aged by surrounding temperature, humidity, or other factors in actual use environments and, therefore, if aging is conducted, physical properties of the rubber itself are degraded and also the interfacial adhesion between reinforcement and rubber are changed more or less. In particular, the temperature of rubber in the tire increases according to the car speed during driving and this can cause the aging of rubber and interfaces. Under normal driving conditions, a surface temperature of tire is up to $125^{\circ} \mathrm{C}$ and the faster driving raises it up to $180^{\circ} \mathrm{C}$. Also, moisture is known as a factor of the aging of the tire rubber. Therefore, the temperature and humid condition has a major effect on the aging of the tire rubber. Such aging of rubber and interface may cause the failure of the tire, and, thus, the research of the aging effects by heat and humidity of the tire rubber is very important [10-15].

In this study, the effects of aging condition on the interfacial properties of the polyketone fiber reinforced natural rubber composite were investigated. Thermal and humidity aging of the polyketone/NR composites were conducted in the limited experimental range for the tire application. The effect of a primer coating for the polyketone fiber on an interfacial adhesion of the polyketone/NR composites was especially evaluated with the aging conditions. Variations of the rubber hardness, interfacial strength, chemical composition of the rubber matrix, and so forth according to the aging conditions were measured and analyzed as well as their morphological changes.

\section{Experimentals}

2.1. Materials. Composition of the natural rubber (NR) used in this study is given in Table 1. Same formulation was used in all experiments. Materials were weighed and mixed in an internal mixer and rolled in a roll mill. Finally, a NR sheet with thickness of $6 \mathrm{~mm}$ was obtained. Polyketone fiber was supplied from " $\mathrm{H}$ " company in Korea. Table 2 shows the properties of the fiber used in this study. Polyketone cord was prepared through the two-for-one twister from polyketone fiber supplied. Twist was in direction S-TPM 868 and direction Z-TPM 434 according to each order.

2.2. Surface Treatment. Polyketone cords were washed with acetone by using ultrasonic agitation for an hour. Then, they were washed with distilled water repeatedly. After purifying polyketone cord, their surfaces were treated with a primer consisting of RFL and epoxy. Typical formulation of the RFL used in this study is given in Table 3. Primer dipping process of the polyketone cord is schematically shown in Figure 1.

2.3. Accelerated Thermal and Humidity Aging. Thermal aging was carried out in an air circulating environmental chamber at temperatures of 105,125 , and $180^{\circ} \mathrm{C}$. Because, under normal driving conditions, a surface temperature of tire is up to $125^{\circ} \mathrm{C}$ and the faster driving raises it up to $180^{\circ} \mathrm{C}$. Aging time was set to 8 days. Data on this phenomenon was presented in the results. For humidity aging, the samples were placed in
TABLE 1: Composition of rubber compound.

\begin{tabular}{lc}
\hline Component & Weight $(\mathrm{g})$ \\
\hline NR & 170 \\
Topping \#1 & 200 \\
ZnO & 35 \\
DP-805 & 1.4 \\
Stearic acid & 7 \\
SRF & 105 \\
Zeosil \#155 & 21 \\
CaCO3 & 400 \\
A \#2 & 56 \\
Anti. Ble-N & 7 \\
Rosin & 21 \\
Acc. DM & 8.4 \\
Acc. CZ & 2.1 \\
\hline
\end{tabular}

TABLE 2: Properties of polyketone fiber used in this study.

\begin{tabular}{lcc}
\hline Fineness (denier) & Strength (g/denier) & Elongation $(\%)$ \\
\hline 747 & 18 & 6.0 \\
\hline
\end{tabular}

TABLE 3: Formulation of the RFL primer used in this study.

\begin{tabular}{lc}
\hline & Wet part (g) \\
\hline RF solution & 16 \\
Resorcinol & 7 \\
Formaldehyde & 1 \\
Sodium hydroxide & 200 \\
Water & 224 \\
Maturation: $25^{\circ} \mathrm{C}, 4 \mathrm{~h}$ & \\
\hline Final dip solution & 224 \\
RF solution & 160 \\
Latex & 5 \\
Ammonium hydroxide & 120 \\
Water & 509 \\
Maturation: $25^{\circ} \mathrm{C}, 12 \mathrm{~h}$ &
\end{tabular}

a humidity chamber for $500 \mathrm{~h}$ at $25,60,85$, and $99 \mathrm{RH} \%$, respectively.

2.4. Surface Characterization. Surface chemical composition of polyketone fiber was analyzed by a XPS (Multilab 2000, Thermo). The XPS spectra were obtained using AlKa ( $h v=$ $1400 \mathrm{eV}$ ) monochromated X-ray source with a voltage of $12 \mathrm{kV}$. Also, morphological changes of polyketone fibers were observed with a scanning electron microscope (SEM, S4700, HITACHI) with surface treatment condition. The hardness of the polyketone cord/NR composites was measured by the Shore type A durometer according to ASTM 2240. All tests were conducted at room temperature.

2.5. Thermal Properties. Using a TGA (TA Instrument), changes in thermal stability of the polyketone fiber/NR composite having thermal and humidity aging were analyzed. 


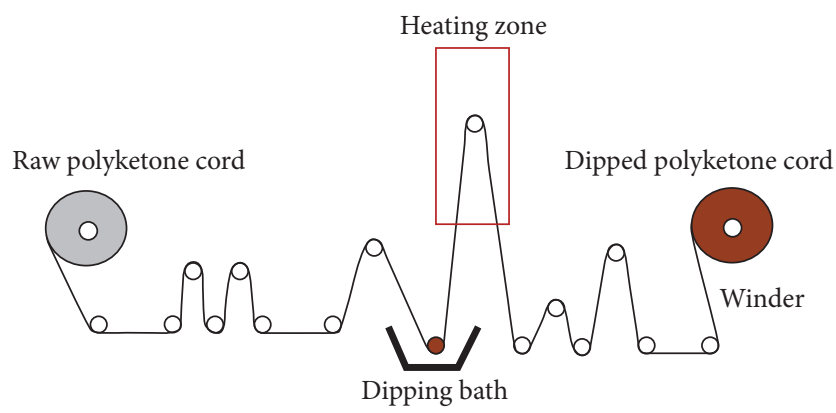

FIGURE 1: Schematics of the primer dipping process.

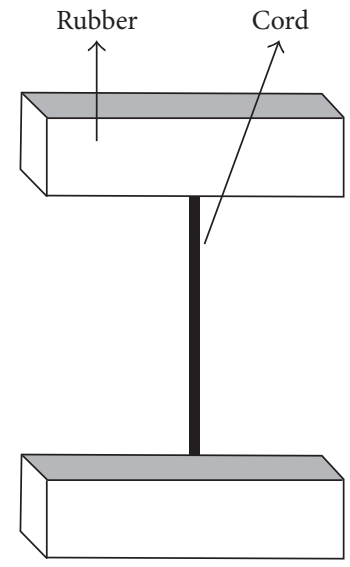

(a)

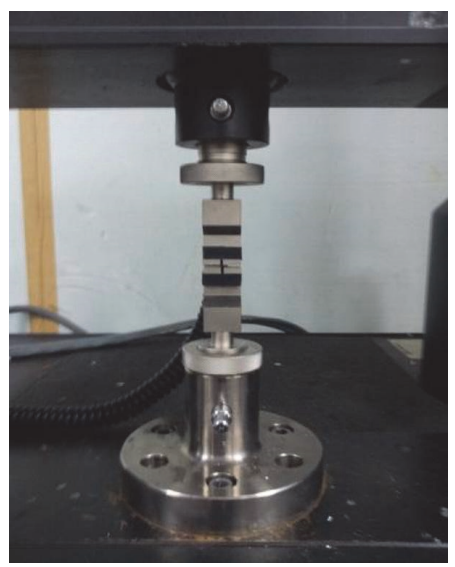

(b)
Figure 2: Preparation of the H-test: (a) the H-test sample; (b) photo of test set-up (ASTM D4776).

Heating rate was $10^{\circ} \mathrm{C} / \mathrm{min}$ from $25^{\circ} \mathrm{C}$ to $800^{\circ} \mathrm{C}$ under nitrogen atmosphere.

2.6. Interfacial Adhesion Test. The interfacial adhesion properties were measured with the $\mathrm{H}$-test method according to ASTM D4776. To prepare the H-test samples, rubber sheets were placed in the channels of a stainless steel mold. Then, the surface treated cord was embedded in rubber sheets. The shapes of the sample and test set-up are shown in Figure 2. Samples were vulcanized at $160^{\circ} \mathrm{C}$, for $30 \mathrm{~min}$ at pressure of $48 \mathrm{MPa}$. Then the products were cut into $\mathrm{H}$-shape samples. Test was performed by using an Instron 4467 tester under room temperature and the cross-head speed was fixed at $125 \mathrm{~mm} / \mathrm{min}$. Adhesion strength was calculated from the maximum load.

\section{Results and Discussion}

3.1. Surface Morphology and Hardness of the Polyketone/NR Composite. To investigate the morphological changes due to aging, surface structure of the polyketone/NR composites was analyzed by the SEM. Figure 3 shows surface morphologies with SEM photograph of the polyketone/NR composite aged under each condition. It is shown that the surface was roughened by thermal aging, and change of the surface occurs more severely at the higher temperature. This phenomenon is due to oxidation and hardening of NR by thermal aging. In general, the cross-linking reaction of the rubber is not the completed reaction. Therefore, a new cross-linking reaction is caused by aging and then the matrix cracks are caused from surface with the progress of the reaction [16]. On the other hand, the change in the surface structure did not occur in the humidity aged sample. Through this, it is expected that oxidation and cross-linking reaction are not caused by humidity aging.

In addition, these characteristics can be quantified as the hardness of the rubber. The hardness of rubber is defined as resistivity of surface for the pressure. Hardness of the rubber is controlled according to the usage of the product and represented as Shore A, D, and OO and number $0 \sim 100$. In the case of a tire, generally Shore $60 \mathrm{~A}$ is the appropriate value. Increase in hardness by aging affects the performance of the product. Figure 4 shows the surface hardness of the polyketone/NR composites thermal aged under $105^{\circ} \mathrm{C}$ and $125^{\circ} \mathrm{C}$. It is shown that hardness increased with aging time. In the case of sample aged at $125^{\circ} \mathrm{C}$, compared to sample aged at $105^{\circ} \mathrm{C}$, the hardness is rapidly increased in the beginning but finally is similar. However, crumbling of surface during the measurement occurred more frequently in the case of sample aged at $125^{\circ} \mathrm{C}$. This phenomenon is because increase of hardness by oxidation occurred more than by cross-linking in the case of sample aged at $125^{\circ} \mathrm{C}$. In addition, the effect of primer on the hardness is not observed. Figure 5 shows the surface hardness of the polyketone/NR composites thermal aged under $180^{\circ} \mathrm{C}$. The increase of hardness was greater than in the other case because of much more oxidation [17]. In addition, in the case of aging temperature of $180^{\circ} \mathrm{C}$, there is no significant difference between sample aged for 2 days and 8 days. It is due to the fast degradation at an early stage of $180^{\circ} \mathrm{C}$ thermal aging followed by the very slow degradation [18]. Also, as with other cases, the effect of primer on the hardness is not observed. Therefore, the processing of the primer has no adverse effects on the physical properties of the NR. However, in the case of the humidity aging, results are different. Figure 6 shows the surface hardness of the polyketone/NR composites aged under each humidity condition. Unlike the results of the thermal aging, change in the hardness by the humidity aging is not observed. Therefore, from the results of SEM observation, it can be said that oxidation and crosslinking reaction are not caused by humidity aging in our experimental range.

3.2. Surface Chemical Composition of the Polyketone/NR Composite. To further investigate the above-mentioned results, the surface chemical composition was analyzed. Table 4 shows functional groups in surface of the polyketone/NR composites. It is shown that $\mathrm{C}-\mathrm{C}$ bond was reduced and $\mathrm{C}=\mathrm{O}$, $\mathrm{COOH}, \mathrm{C}-\mathrm{OH}$, and $\mathrm{O} 1 \mathrm{~s}$ increased in the case of thermal aged specimens. This result is due to oxidation by thermal aging. When $\mathrm{C}-\mathrm{C}$ bonds are broken by thermal energy during thermal aging, carbon radicals are formed. In the presence of atmospheric oxygen, the functional groups containing oxygen such as hydroperoxide are formed from reaction of 


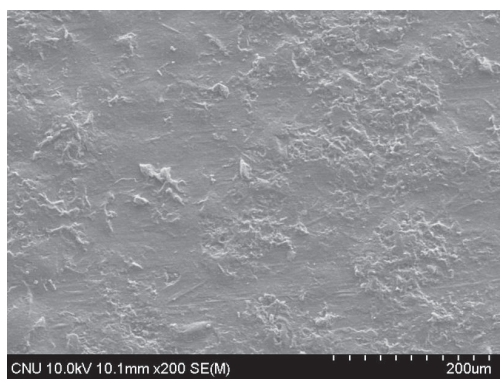

(a)

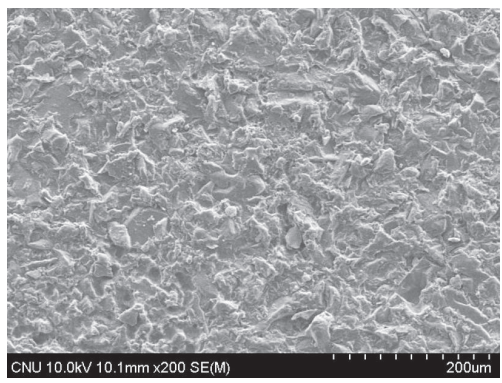

(d)

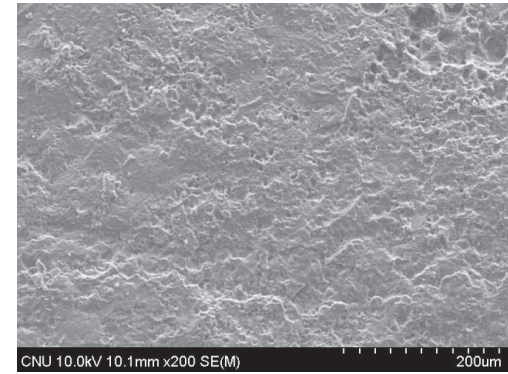

(b)

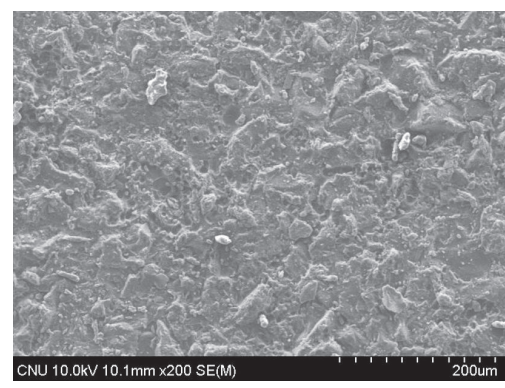

(e)

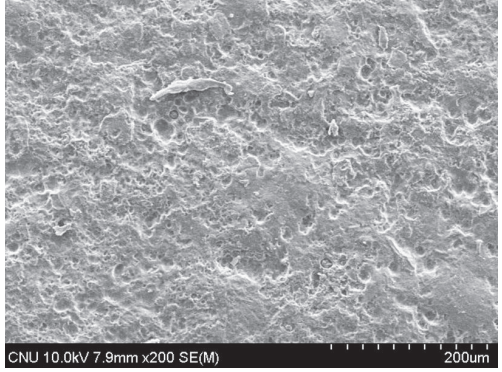

(c)

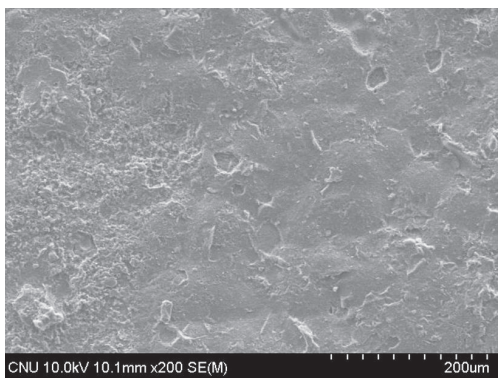

(f)

FIGURE 3: SEM photographs of polyketone/NR composite surface; (a) nonaged, (b) thermal aged at $105^{\circ} \mathrm{C}\left(8\right.$ days), (c) $125^{\circ} \mathrm{C}(8$ days), (d) $180^{\circ} \mathrm{C}$ (2 days), and (e) $180^{\circ} \mathrm{C}$ (8 days), and (f) humidity aged at $99 \%(500 \mathrm{~h})$.

TABLE 4: Surface functional groups analyzed by XPS of the polyketone/NR composite as thermal aging and humidity aging.

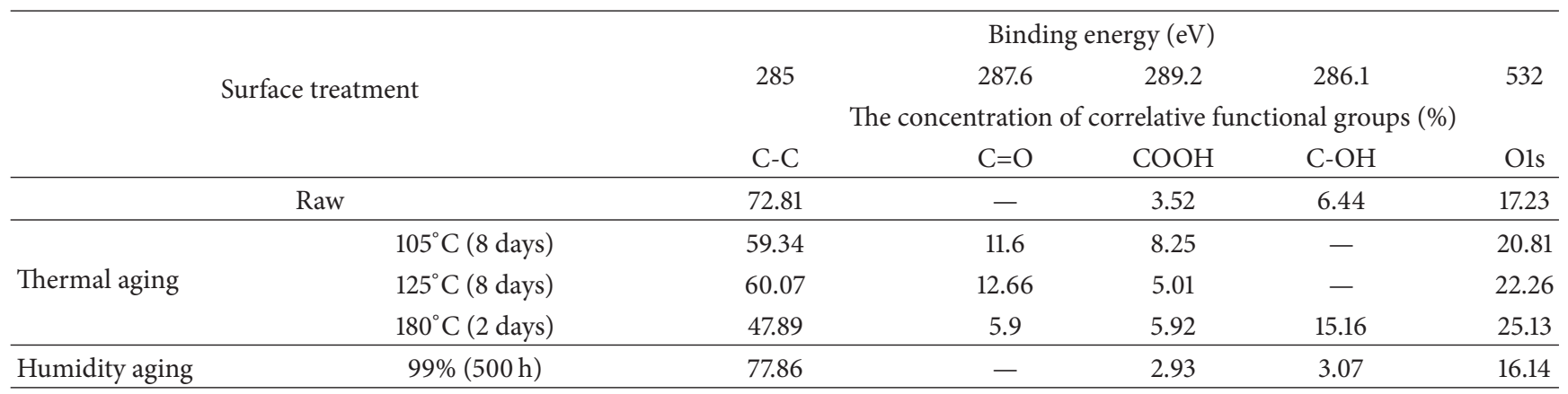

carbon radical [19]. In all conditions of the thermal aging, these phenomena appear to be identical to each other; the oxidation occurs more severely with an increase in aging temperature. However, in the case of humidity aging, this phenomenon does not occur since there is no oxidation. Therefore, the analysis result of humidity aged sample is substantially the same as the raw sample.

Table 5 shows the results of XPS surface composition analysis. Similar conclusion can be obtained through this result. It can be seen that the oxygen content increased due to oxidation. But, in the case of humidity aging, the oxidation does not occur, because the decomposition of the mainchain does not occur since thermal energy is not applied. In addition, in the case of aging temperature of $180^{\circ} \mathrm{C}$, there is no significant difference between sample aged for 2 days and for 8 days. This is because most of the oxidation is completed at an early stage by high temperature and subsequent aging proceeds very slowly.
TABLE 5: Chemical composition of the polyketone/NR composite as thermal aging and humidity aging.

\begin{tabular}{|c|c|c|c|c|}
\hline \multicolumn{2}{|c|}{ Surface treatment } & \multicolumn{2}{|c|}{$\begin{array}{c}\text { Chemical composition } \\
\text { (\%) }\end{array}$} & \multirow{2}{*}{$\begin{array}{c}\text { Atomic } \\
\text { ratio } \\
\mathrm{O} / \mathrm{C}\end{array}$} \\
\hline & & $\mathrm{C}$ & $\mathrm{O}$ & \\
\hline & Raw & 82.77 & 17.23 & 0.208 \\
\hline \multirow{4}{*}{$\begin{array}{l}\text { Thermal } \\
\text { aging }\end{array}$} & $105^{\circ} \mathrm{C}$ (8 days) & 79.19 & 20.81 & 0.262 \\
\hline & $125^{\circ} \mathrm{C}$ (8 days) & 77.74 & 22.26 & 0.286 \\
\hline & $180^{\circ} \mathrm{C}$ (2 days) & 74.87 & 25.13 & 0.335 \\
\hline & $180^{\circ} \mathrm{C}$ (8 days) & 74.44 & 25.56 & 0.343 \\
\hline $\begin{array}{l}\text { Humidity } \\
\text { aging }\end{array}$ & $99 \%(500 \mathrm{~h})$ & 83.86 & 16.14 & 0.192 \\
\hline
\end{tabular}

3.3. Thermal Properties of the Polyketone/NR Composite. To investigate the changes in thermal properties of polyketone/NR composite by aging, TGA analysis was carried out. 


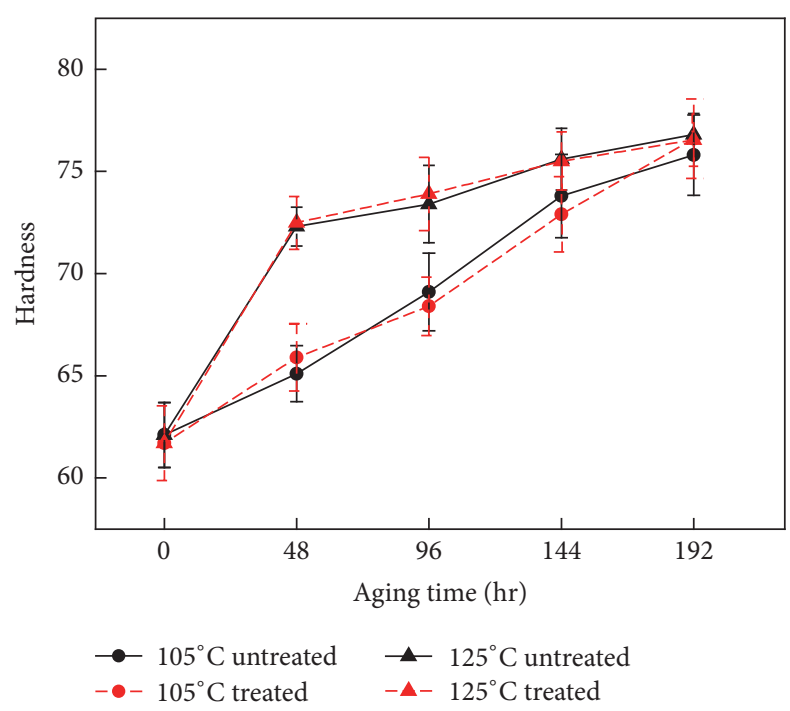

FIGURE 4: Hardness of the polyketone/NR composite after thermal aging at 105 and $125^{\circ} \mathrm{C}$.

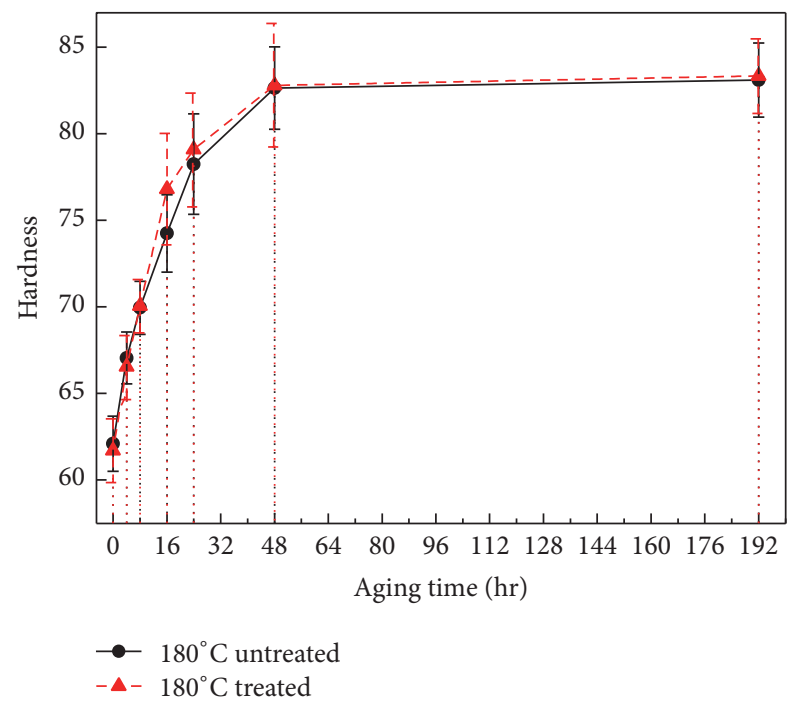

Figure 5: Hardness of the polyketone/NR composite after thermal aging at $180^{\circ} \mathrm{C}$.

Figure 7 shows TGA and DTG curves of the polyketone/NR composites aged under each temperature. Thermal decomposition peak appears prominently at $370 \sim 450^{\circ} \mathrm{C}$. But in the case of raw sample and aged sample at 105 , thermal decomposition peak at $450^{\circ} \mathrm{C}$ appears most significantly, while in the case of sample aged at $125^{\circ} \mathrm{C}$ and $180^{\circ} \mathrm{C}$, peak at $370^{\circ} \mathrm{C}$ appears most significantly. This difference in decomposition behavior is due to the difference in aging mechanism. In general, the mechanism of thermal aging is classified into two types. One is due to cross-linking and the other is due to chain degradation. If thermal aging is conducted at temperature higher than $120^{\circ} \mathrm{C}$, aging caused by chain degradation becomes more active by oxidation [19]. Therefore, in the case of sample aged at $125^{\circ} \mathrm{C}$ and $180^{\circ} \mathrm{C}$, peak is at $450^{\circ} \mathrm{C}$ which means the mainchain decomposition appears smaller than the case of sample

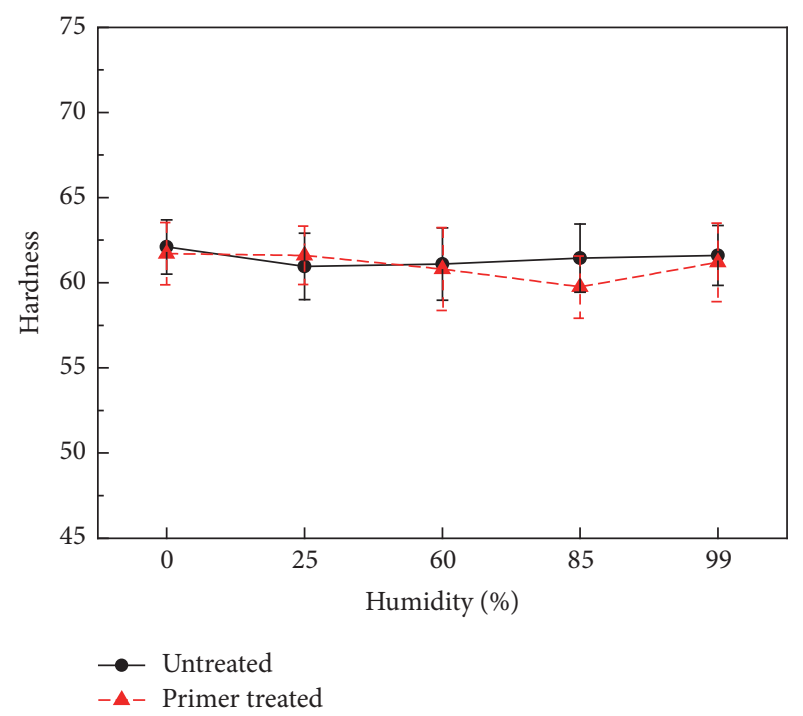

FIGURE 6: Hardness of the polyketone/NR composite after humidity aging.

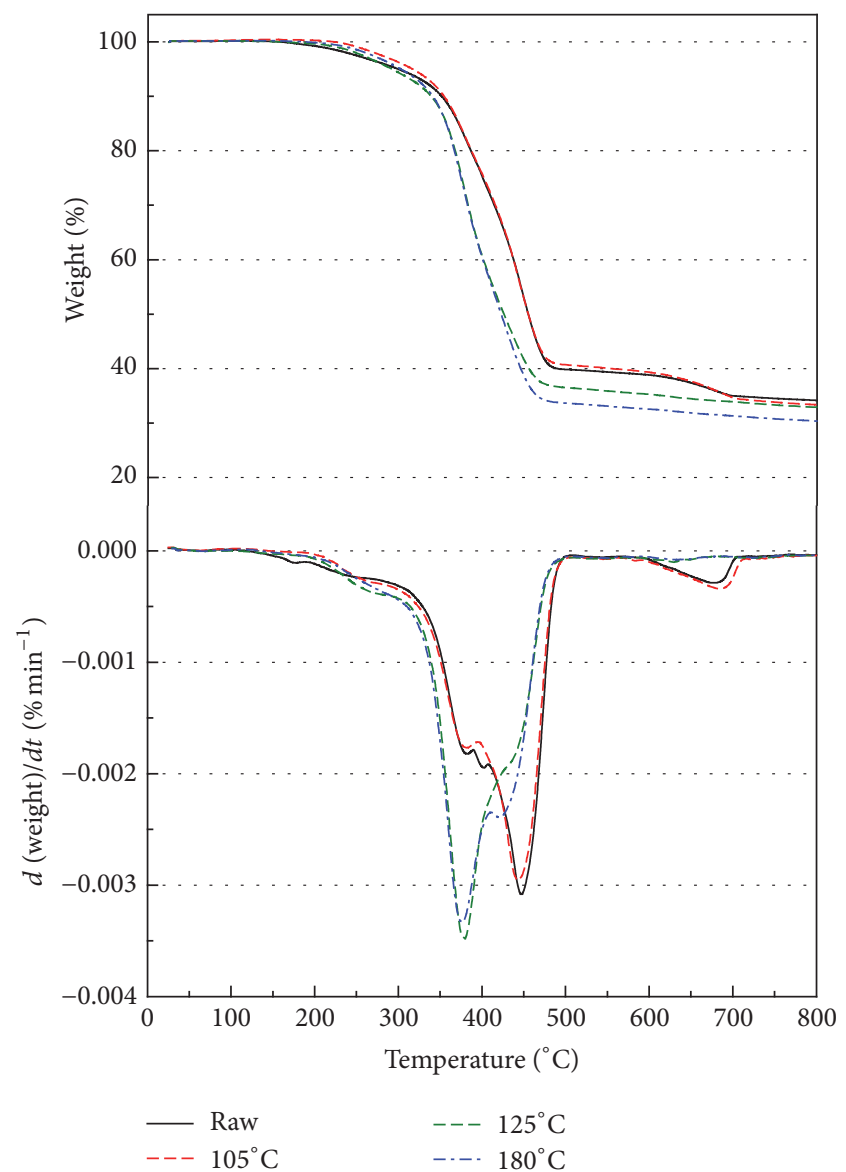

FIGURE 7: TGA and DTG curve of thermal aged polyketone/NR composites.

aged at $105^{\circ} \mathrm{C}$. This difference in aging mechanism appears to be the difference in increase rate of the initial hardness in the previous analysis results of the hardness. 


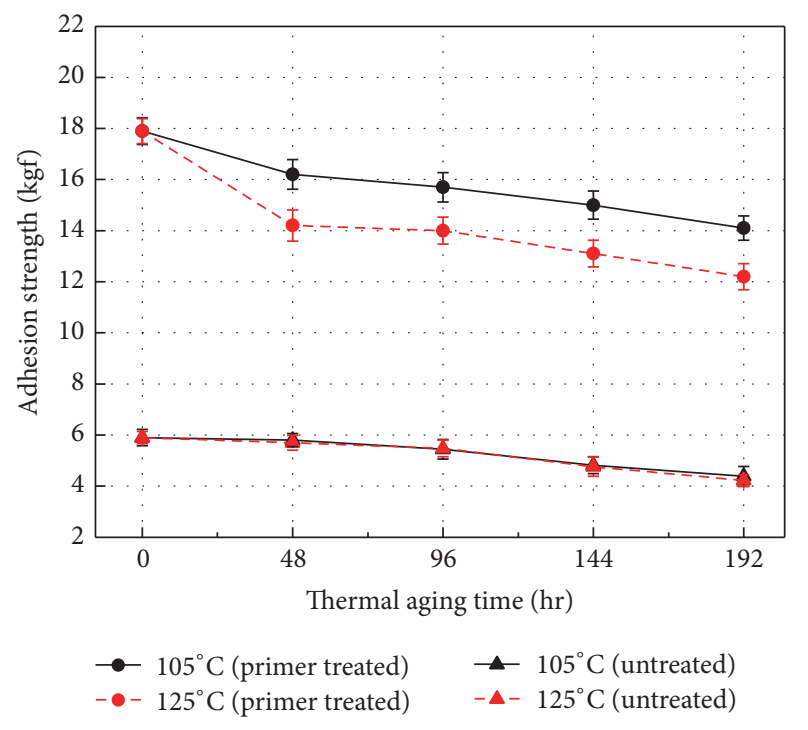

FIgURE 8: Adhesion properties of thermal aging at 105 and $125^{\circ} \mathrm{C}$ with and without primer treatment.

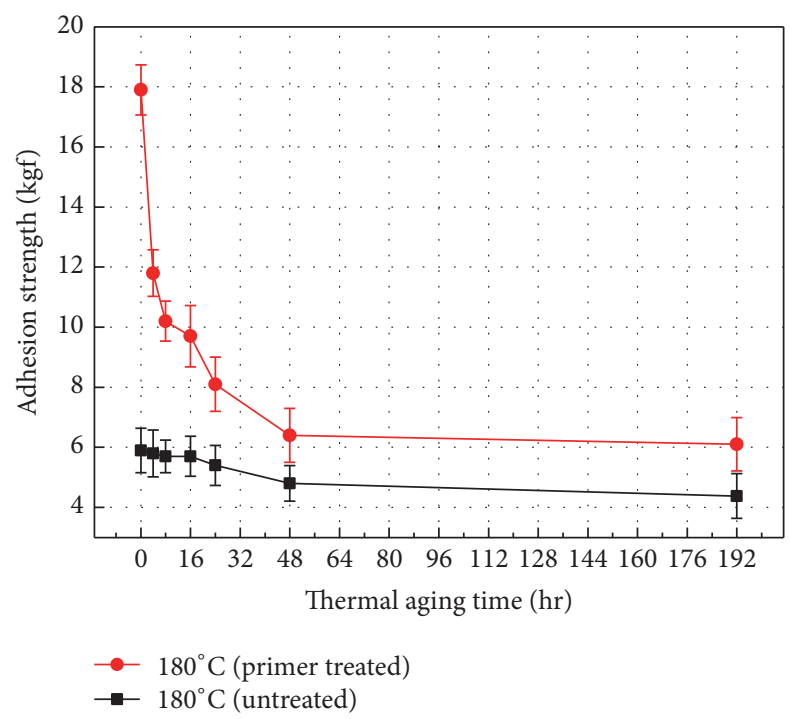

FIGURE 9: Adhesion properties of thermal aging at $180^{\circ} \mathrm{C}$ with and without primer treatment.

3.4. Interfacial Properties of the Polyketone/NR Composite. To investigate the change in interfacial adhesion strength of the polyketone/NR composite by primer treatment and aging, $\mathrm{H}$ test was carried out. Figures 8 and 9 show the effect of thermal aging on interfacial adhesion strength of the polyketone/NR composites with a primer treatment. The adhesion strength increased largely, almost three times, by a primer treatment compared to untreated case. Therefore, primer coating is very effective in high surface adhesion between polyketone cord and rubber matrix. Also, adhesion strength in all samples decreased by thermal aging. However, adhesion strength of the primer coated cases decreased more than noncoated cases by thermal aging. This result is due to oxidation of NR during thermal aging and the reason of this phenomenon can be verified through observing adhesion fracture surfaces. Figure 10 shows SEM photographs in adhesion fracture surface of the primer treated and untreated polyketone/NR composite aged under each temperature. In the case of untreated composite, the NR is not properly impregnated into the fiber due to a lack of compatibility. Therefore, the interfacial adhesive strength is weak and the rubber residues are not left on the fiber surface. However, in the case of treated composites, the NR is well impregnated into the fiber and there remain rubber residues on the fracture surface. Rubber residues are observed to be thicker at higher aging temperatures. These results mean that most of the fracture is generated from the rubber region weakened by oxidation. Thus it can be considered that although rubber matrix degraded by oxidation at thermal aging, its interfacial region, caused by a primer coating, was not degraded largely by a protection effect of primer. Nevertheless, finally at highest temperature and long aging time, interfacial strength of the composite decreased largely due to the matrix degradation. Also, in the case of aging temperature of $180^{\circ} \mathrm{C}$, there is no significant difference between sample aged for 2 days and 8 days as in the other analysis results. It is due to the fast degradation at an early stage of $180^{\circ} \mathrm{C}$ thermal aging followed by the very slow degradation [20].

Figure 11 shows the effect of humidity aging on the interfacial strength of the polyketone/NR composites with a primer treatment. However, change in the interfacial strength under the humidity aging is not observed, because the decomposition of the main-chain does not occur when high thermal energy is not applied, and, thus, it means that oxidation is not caused by humidity aging. And infiltration of moisture into the matrix and interface does not occur in our experimental humidity-time range [21].

\section{Conclusions}

The effects of thermal and humidity aging on the characteristics of the polyketone fiber reinforced natural rubber composite were investigated and analyzed in accordance with the primer coating of fiber. The rubber hardness of the polyketone/NR composite increased with increasing thermal aging time because of the oxidation and the cross-linking reaction of the rubber matrix. Oxygen content of the NR matrix increased with the thermal aging temperature and time due to the surface oxidation at an elevated temperature. Lots of oxygen containing groups were increased by thermal aging. Oxidation of the NR matrix by thermal aging caused cracked and roughened morphologies and it brought the decrease of the interfacial strength of the composites. In addition, the oxidation occurred more actively in the aging temperature above $125^{\circ} \mathrm{C}$. In the cases of using the primer treated polyketone fiber, the composites have higher adhesion strength than the untreated one. Strong adhesion between the primer treated polyketone fiber and NR matrix protected the degradation of the interfacial strength against thermal aging. On the other hand, aging of the polyketone/NR composites under various humidity conditions did not have a significant impact on the interfacial adhesion and hardness. Therefore, it can be considered that moisture condition has almost no influence on the polyketone/NR composite interface in our experimental humidity-time range. 


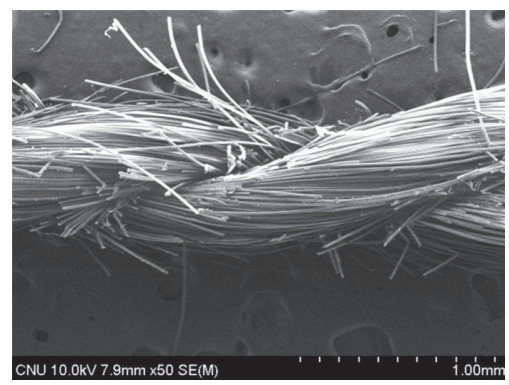

(a)

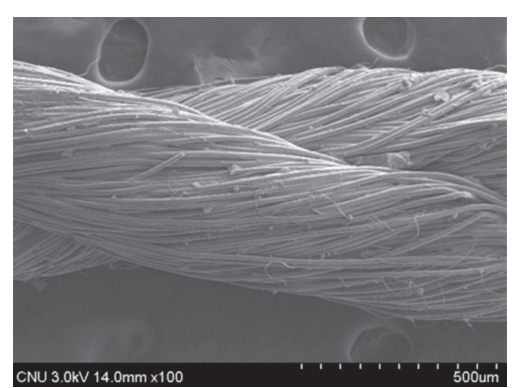

(b)

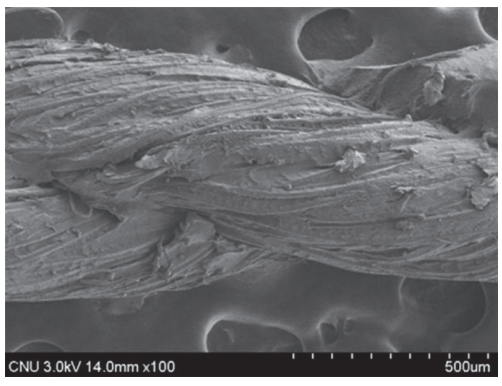

(c)

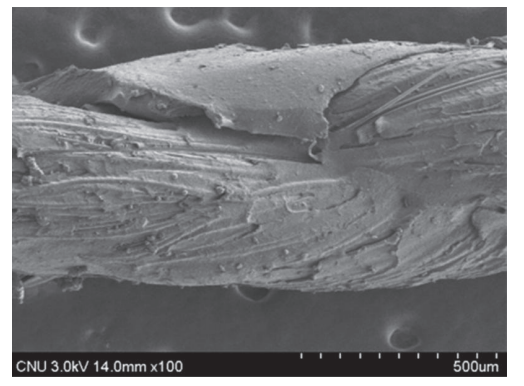

(d)

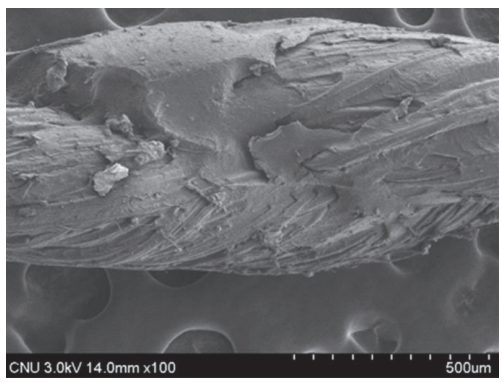

(e)

FIGURE 10: SEM photographs of adhesion fracture surface of polyketone/NR composites; (a) primer untreated and nonaged, (b) primer treated and nonaged, and (c) primer treated and thermal aged at $105^{\circ} \mathrm{C}$, (d) $125^{\circ} \mathrm{C}$, and (e) $180^{\circ} \mathrm{C}$.

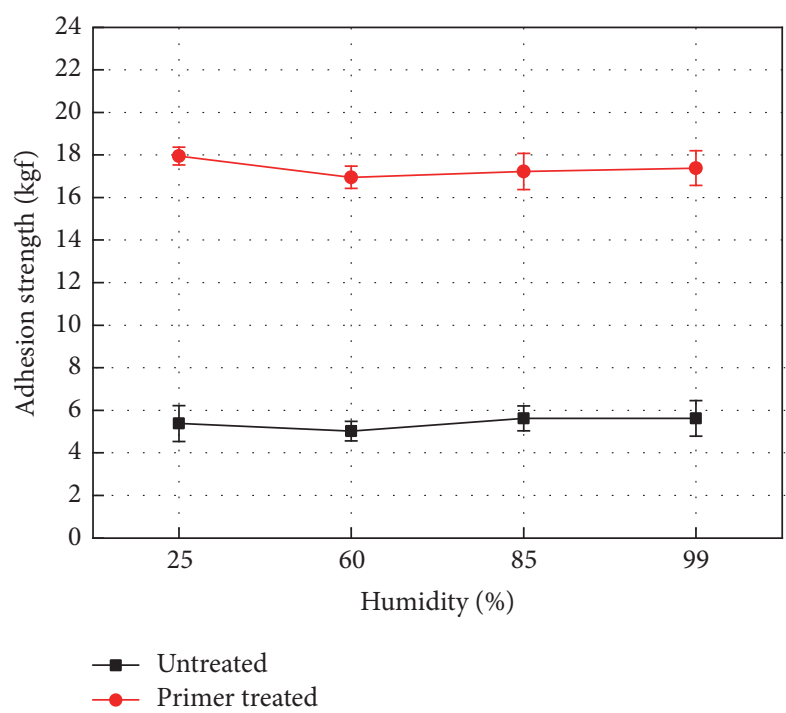

FIGURE 11: Adhesion properties of humidity aging at 25, 60, 85, and 99.

\section{Competing Interests}

The authors declare that they have no competing interests.

\section{Acknowledgments}

This research was financially supported by the "Fundamental R\&D Program for Technology of the Graduate Student Education Program for Research of Hybrid and Super Fiber
Materials" through the Ministry of Trade, Industry \& Energy (MOTIE) and the Korea Institute for Advancement of Technology (KIAT) (N0000993).

\section{References}

[1] D. K. Setua and S. K. De, "Short silk fiber reinforced natural rubber composites," Rubber Chemistry and Technology, vol. 56, no. 4, pp. 808-826, 1983.

[2] L. A. Goettler and K. S. Shen, "Short fiber reinforced elastomers," Rubber Chemistry and Technology, vol. 56, no. 3, pp. 619638, 1983.

[3] Y. Zuigyo and M. Yamamoto, "Basic study of PK fiber tire cord," Tire Science and Technology, vol. 35, no. 4, pp. 317-325, 2007.

[4] M. Jamshidi, F. Afshar, N. Mohammadi, and S. Pourmahdian, "Study on cord/rubber interface at elevated temperatures by $\mathrm{H}$ pull test method," Applied Surface Science, vol. 249, no. 1-4, pp. 208-215, 2005.

[5] A. K. Naskar, A. K. Mukherjee, and R. Mukhopadhyay, "Studies on tyre cords: degradation of polyester due to fatigue," Polymer Degradation and Stability, vol. 83, no. 1, pp. 173-180, 2004.

[6] T. S. Solomon, "Systems for tire cord-rubber adhesion," Rubber Chemistry and Technology, vol. 58, no. 3, pp. 561-576, 1985.

[7] M. Jamshidi and F. A. Taromi, "Estimation of dynamic adhesion from static test results in the model cord-RFL-rubber system," Journal of Adhesion Science and Technology, vol. 20, no. 15, pp. 1693-1703, 2006.

[8] R. B. Durairaj, "Resorcinol formaldehyde latex (RFL) adhesives and applications," in Resorcinol: Chemistry, Technology and Applications, pp. 263-339, Springer, 2005.

[9] W. B. Wennekes, Adhesion of RFL-Treated Cords to Rubber: New Insights into Interfacial Phenomena, University of Twente, 2008. 
[10] W. J. van Ooij, "Mechanism and theories of rubber adhesion to steel tire cords-an overview," Rubber Chemistry and Technology, vol. 57, no. 3, pp. 421-456, 1984.

[11] N. Rattanasom, A. Poonsuk, and T. Makmoon, "Effect of curing system on the mechanical properties and heat aging resistance of natural rubber/tire tread reclaimed rubber blends," Polymer Testing, vol. 24, no. 6, pp. 728-732, 2005.

[12] A. K. Naskar, S. K. De, A. K. Bhowmick, P. K. Pramanik, and R. Mukhopadhyay, "Characterization of ground rubber tire and its effect on natural rubber compound," Rubber Chemistry and Technology, vol. 73, no. 5, pp. 902-911, 2000.

[13] J. M. Baldwin and D. R. Bauer, "Rubber oxidation and tire aging- a review," Rubber Chemistry and Technology, vol. 81, no. 2, pp. 338-358, 2008.

[14] Y. Iyengar, "Factors in rubber compounds affecting the adhesion of polyester tire cords," Journal of Applied Polymer Science, vol. 15, no. 2, pp. 267-276, 1971.

[15] M. Forrest, Long-Term and Accelerated Ageing Tests on Rubbers, vol. 110, iSmithers Rapra Publishing, 2000.

[16] D. Huang, B. J. LaCount, J. M. Castro, and F. Ignatz-Hoover, "Development of a service-simulating, accelerated aging test method for exterior tire rubber compounds I. Cyclic aging," Polymer Degradation and Stability, vol. 74, no. 2, pp. 353-362, 2001.

[17] A. Tóth,, I. Bertóti,, M. Blazsó, G. Bánhegyi, A. Bognar, and P. Szaplonczay, "Oxidative damage and recovery of silicone rubber surfaces. I. X-ray photoelectron spectroscopic study," Journal of Applied Polymer Science, vol. 52, no. 9, pp. 1293-1307, 1994.

[18] E. J. Blackman and E. B. McCall, "Relationships between the structures of natural rubber vulcanizates and their thermal and oxidative aging," Rubber Chemistry and Technology, vol. 43, no. 3, pp. 651-663, 1970.

[19] J. I. Cunneen, "Oxidative aging of natural rubber," Rubber Chemistry and Technology, vol. 41, no. 1, pp. 182-208, 1968.

[20] V. G. Geethamma, G. Kalaprasad, G. Groeninckx, and S. Thomas, "Dynamic mechanical behavior of short coir fiber reinforced natural rubber composites," Composites Part A: Applied Science and Manufacturing, vol. 36, no. 11, pp. 1499-1506, 2005.

[21] A. Hodzic, J. K. Kim, A. E. Lowe, and Z. H. Stachurski, "The effects of water aging on the interphase region and interlaminar fracture toughness in polymer-glass composites," Composites Science and Technology, vol. 64, no. 13-14, pp. 2185-2195, 2004. 

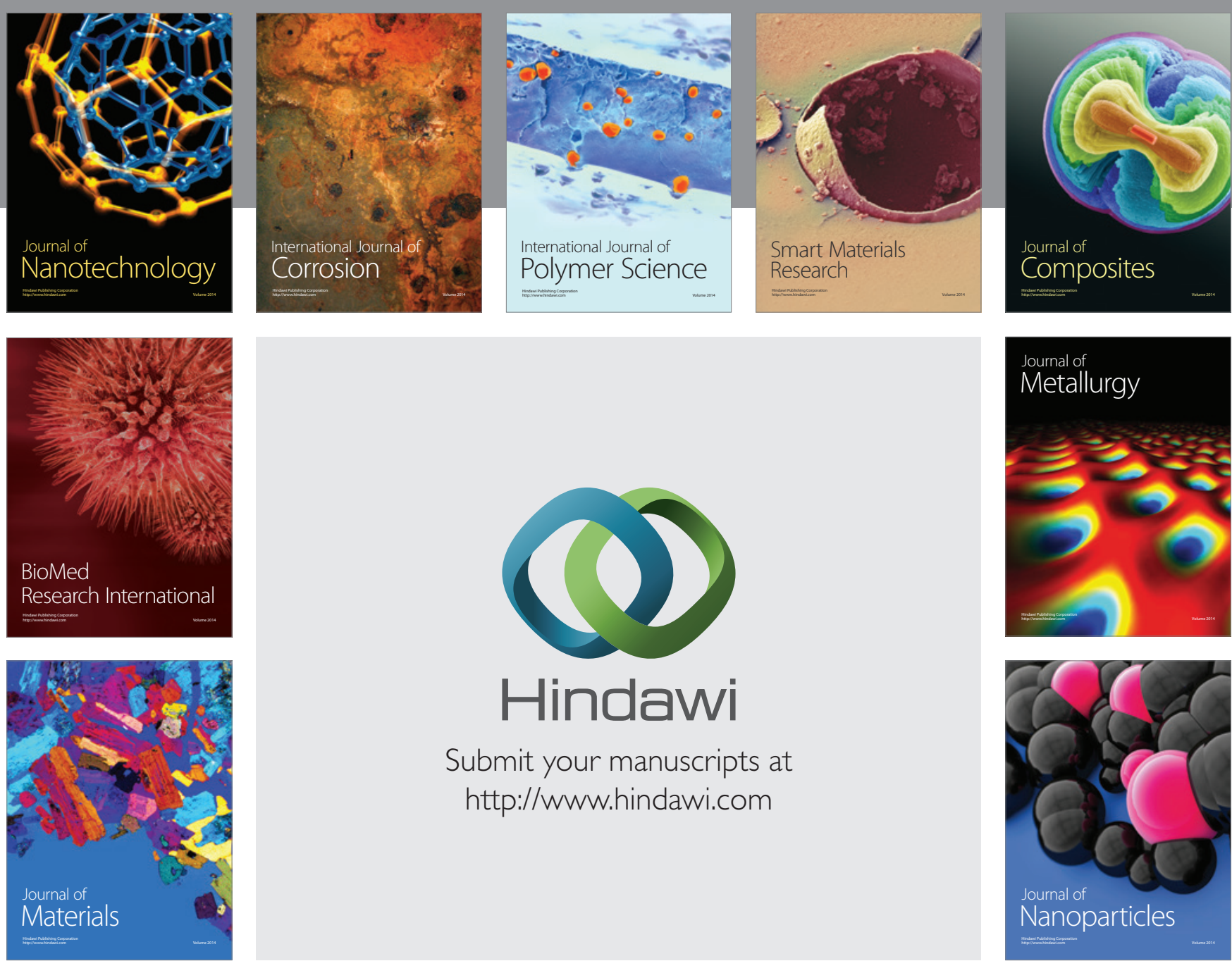

\section{Hindawi}

Submit your manuscripts at

http://www.hindawi.com

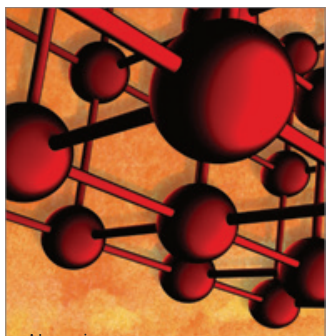

Materials Science and Engineering
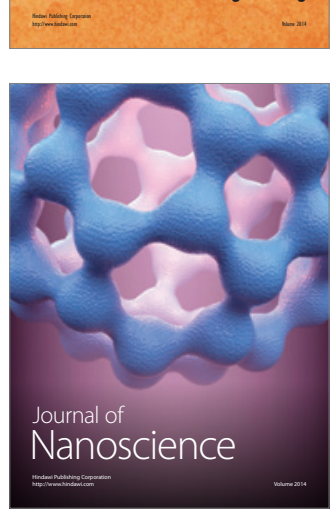
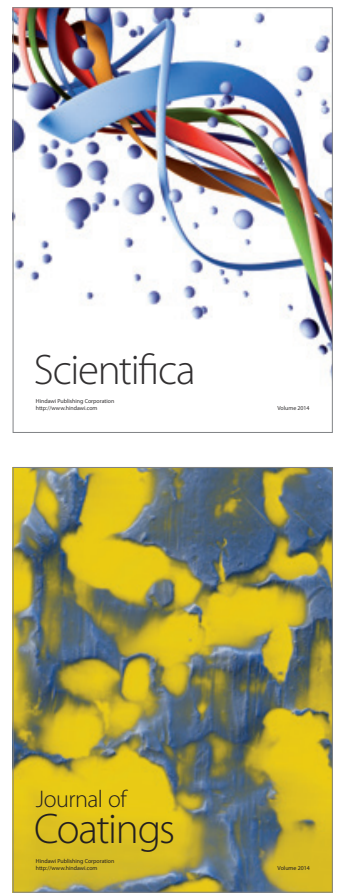
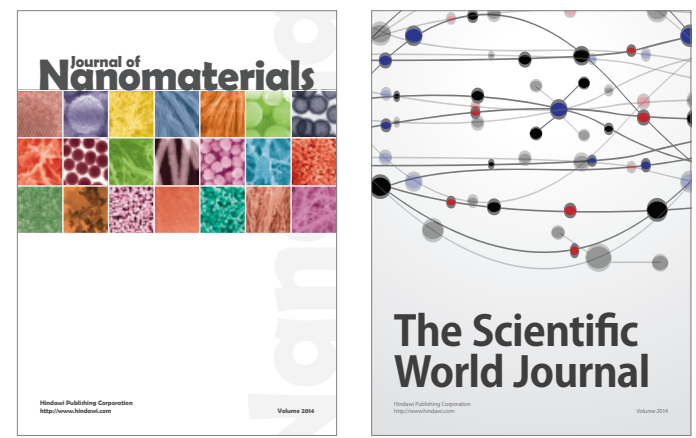

The Scientific World Journal
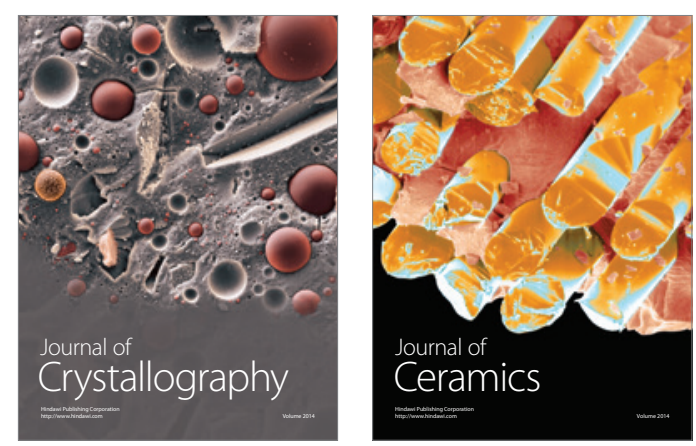
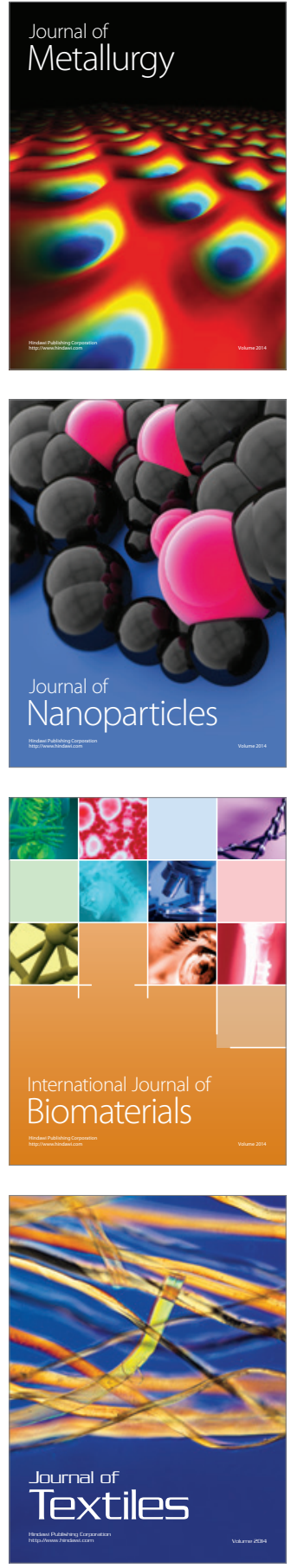